\title{
3D Animation: Don't Drink and Drive
}

\author{
Imran Ahmed and Satish Janghel \\ SCSE, VIT University, Vellore, Tamil Nadu-632014 \\ imranahmed2k9@gmail.com,satishjanghel@gmail.com
}

\begin{abstract}
A 3D human model, car, building, trees are developed using blender, make Human, lightwave and 3Dstudio. Aim of the paper is to animate don't drink drive case using $3 D$ modeling. A human model is drunk with the surrounding of buildings and trees, sits in car and met with accident after striking with the trees. This paper gives message not to drink while driving. An animation is developed open source python based blender and human model is developed using make Human and is utilize by blender software and objects are used by blender as it supports all types of format like lwo,bvh,3ds,mhx,c3d,c4d and the animation consist of 168 frames with timeline of 10 seconds.
\end{abstract}

Keywords: 3D human model, Make Human, 3D Studio, Blender, Python

\section{Aim}

Aim of this paper is "Do not drive after drinking". 3D animation is developed by showing how a human model meets with an accident after consuming alcohol. So, this animation will be helpful in displaying the aim of the paper.

\section{Motivation}

After seeing and reading many accident stories on television and newspaper, a need of motivating people about the accidents taking place after drinking and also meeting accident with innocent people, results in the loss of human lives. As a result, this animation try to motivate people not to drive after drinking.

\section{Introduction}

3D is defined as any object that occurs on 3 axis ( $\mathrm{x}, \mathrm{y}$ and $\mathrm{z}$ axis) based on Cartesian coordinate system. In $x$-axis, being represented horizontal and $y$-axis vertical, but in 3D there is another third axis (z-axis) which represent depth. 3D models are represented by using 3D object in the collection of points at 3D space. It can be connected by various geometric entity such as triangles, lines, curved surface, etc. In computer modelling, the representation of 3 dimensional objects on a computer, using some form of software that is designed for the 3D modeling purposes.

Animation is the display of sequence of images in the 3D space so as to create an illumination of movement. Its effects in image should be realistic, so that it's easy to understand to the user. 3D animation is the computer generated that gives object space. In 3D, the images and the graphics uses 3 dimension which gives the characters depth and presentations, which looks real and living. The graphics designer is designed by using the different types of software for representation of pictures and images. There are various types of software to represent the 3D animations. These are Autodesk 3ds Max, AutoCAD, 
Blender, LightWave 3D, Poser, Sketchup. But, this paper uses Blender and Make Human software for 3D animation.

This paper "3D Animation: Don't Drink and Drive" is modelled by using Blender and Make Human software. A human is made by using Make Human software and by using Blender, a car and a tree is made. The person is drunk, he drives the car and met with an accident with the tree. This whole scenario is shown by 3D animation video. And we get a lesson not to drink and drive.

3D modelling animation gives graphically simplified complicated concepts and convey the complex inter-relationships between the objects which are difficult to visualize. Concepts and Ideas which are not represented in words, it can be easily created by Animation. It captures the attention to the user and the information which is presented as a moving image is returned by the user for a longer time and accurately. Animation can recreate the event.

\section{Literature Survey}

2-dimensional animation is mostly based upon the transformation of the images both including images and videos. Transformation [9] is used by developer to resize, reposition the model [1-5] without any change in the base value. Other thing is that in $2 \mathrm{~d}$ animation [2] concatenating together sequences of captured video [6]. If it follows traditional animation it takes more time than the computer animation [2], for a large number of drawing and photographs for completing the production. And it is very difficult to speed up the production as each animator because he works at a different speed. Traditional animation takes more time compared to the computer animation.

Because computer animation uses animation software [7-8] and speeds up the performance [13]. If a small mistake is found in traditional amount the animation is required to repeat from start, instead of deleting and correcting mistake so again the same work can be done, so there is time consuming in traditional animation. For more accuracy large number of task are to be repeated for correctness so it is time consuming and cost also increase. The traditional animation requires the equipment and different type of tools [14] for animation, such as tracing tool [11-12], photographic tool; artistic tool etc. Traditional animations lack the market interest because it uses wide range of special artist tools. Very large rooms are required in the entire process from drawing to tracing and then $t$ to the photography. Then the $2 \mathrm{~d}$ animation is developed which is easy to design but does not looks exactly realistic image and is cheaper in nature.

2D animation [5] is not based upon the transformation of images so then new concept is coming 3Danimation [3, 10, 13] which is uses the concept of translation, scaling, rotation etc. $3 \mathrm{~d}$ models are connected to the various geometric entities likes triangles, lines curved surfaces etc.it can be created by using concepts like scanned method and hand algorithm[6]. 3d animation [3]- 4, 9] can give better realistic images so this study uses 3D animation for modeling a human, a car, a tree using the concept of drink and drive. Person drinks and drives a car; due to this the car is in an accident with the tree. By the help of the 3D animation, it can state the concept "do not drink and drive" [15]. This animation can graphically simplify the complex concept and convey the interrelationship which is difficult to visualize [16]. Some concepts and ideas which cannot be represented in words, but can be represented in the form of animation. Animation can combine large amount of scientific data [17] in to a compact package, which can be represented easily. Animation captures attention and information which is presented as a moving image is retained by the viewer for longer amount of time and gives most accurately .virtual light sources from different angels are used 
with reflection. This paper uses the make human and blender [4] software with different files is used.

\section{Concepts Used}

\subsection{Modeling}

Normally all modeling starts with cube modeling and moves further to the moving in and out the vertices of the cube to make larger or smaller and complicated structure or mesh. Another is modeling by curves which gives us objects like wall and flat surfaces. Modeling can be performed by using various primitives available with blender like

5.1.1. Meshes: Whole object is converted into a form of mesh such that a surface of object can be change by just pinching in and out the surface of object.

5.1.2. Curves: These are used to model the objects outline and it provide functionality by controlling the outline of the object.

5.1.3. Surfaces: It provide a way to for modeling the NURBS surface. NURBS surface are controlled by weighted points chosen on the surface.

5.1.4. Text: It provides an efficient way for displaying $3 \mathrm{D}$ text in the $3 \mathrm{D}$ space for proving textual information to the viewer.

5.1.5. Duplication Objects: It is most important tool for repetition of particular objects in the 3D space.

\subsection{Modifier and Deformation}

Modifiers are based on automatic operation so that it affects an object. By help of modifier an animator can perform many effects automatically; it is very difficult do manually and without change of base topology of the object. Modifier works by changing how an object is displayed and rendered but not told about the actual geometry what it is used. Several modifier s are added to a single object to form a modifier stack. Modifier is applied to make changes the permanent.

5.2.1 Modify: Modify is a group of modifiers which is not directly affected the shape of the object and may affect some other data like vertex.

Mesh cache: It is used apply for animated mesh data to a mesh.

UV project: project UV coordinates from the mesh.

UV Wrap: It can be helpful for dynamically edit the UV coordinates on the mesh data.

Vertex Weight: It is used for edit a group of vertex in a mesh data.

5.2.2. Generate: Generate is a group of modifier are used for either change the general appearance or automatically add new geometry to an object.

Array: It creates an array of mesh and similar shapes.

Bevel: It creates a bevel on a selected mesh object.

Build: it arranged the mesh step by step at the time of animating.

Decimate: By help of this number of polygon count of mesh reduced.

Mask: it is used to hide some parts of the mesh.

Mirror: mirror is an object about its own axis so that gives the resultant mesh of symmetrically.

Wireframe: it converts all faces to a wireframe. 
5.2.3. Deform: Deform is a group of modifier are used change the shape of object and sometimes used for changing the curves, surfaces.

Armature: it is used in bones for deform purposes and that help for animated object.

Cast: Suppose changing the shape of mesh, surface or lattice of a sphere.

Wave: it is used to deform the object.

5.2.4. Simulate: The simulate group of modifiers to activate simulations. These modifiers are automatically added to the modifiers stack.

\subsection{Lighting}

It plays a vital role in rendering a modeled object for viewing to viewer. The modeled object and surface will give bad viewership to viewer but with lighting a simple object becomes more realistic and close to world real object. Color of objects and lighting will be affected by following reasons as follows:

1. One's visual ability to distinguish between colors.

2. The mode of medium through an image is viewed.

3. The level of quality of image provides.

4. The surrounding and environments in which an image or object is seen.

5. Human brain color perception and intensity of object relative to its surrounding.

6. Color of ambient light of world viewport.

7. The way ambient light is made available to object by use of occlusion ambient.

8. Indirect lighting by which an object covers another object by its color.

\subsection{Shadows}

The complement of light is called shadow. It is like reducing or removing the intensity of source of light from highlighting or illuminating the object. It provide contrast to object and no object exists in real where shadows is not there, it is like shadows never leaves us even after death, shadows always exist for an object. Shadows can be subdivided into other categories like Ray Traced lamp Shadows, Buffer lamp Shadows, Ambient occlusion, Indirect lighting. Shadows can occur like an object which is opaque can block light on falling on object.

\subsection{Materials}

It defines the material quality of the substance of which an object is made. An object is made of is reflected by the color of an object, shininess of an object and reflective property of object as well as it also show the how opaque or transparent an object is, and how diffusion takes place through the object. Material of an object can be like gold, silver, wood, steel or any other thing like clay. One must should have understanding of how material and light interact inside render engine. The image which is rendered by render engine is the projection of a given scene onto a virtual or say imaginary plane called as viewing plane. For rendering an image of a given scene one must decide from which direction of 3D space light is falling at each pixel of given plane. The easiest and most simple way is to follow backwards from the pixel where light is falling to the original source of light. The properties of given surface and the light falling or angle of incident light always let us know what amount of light will be reflected backwards along the angle of viewing plane incidences. 


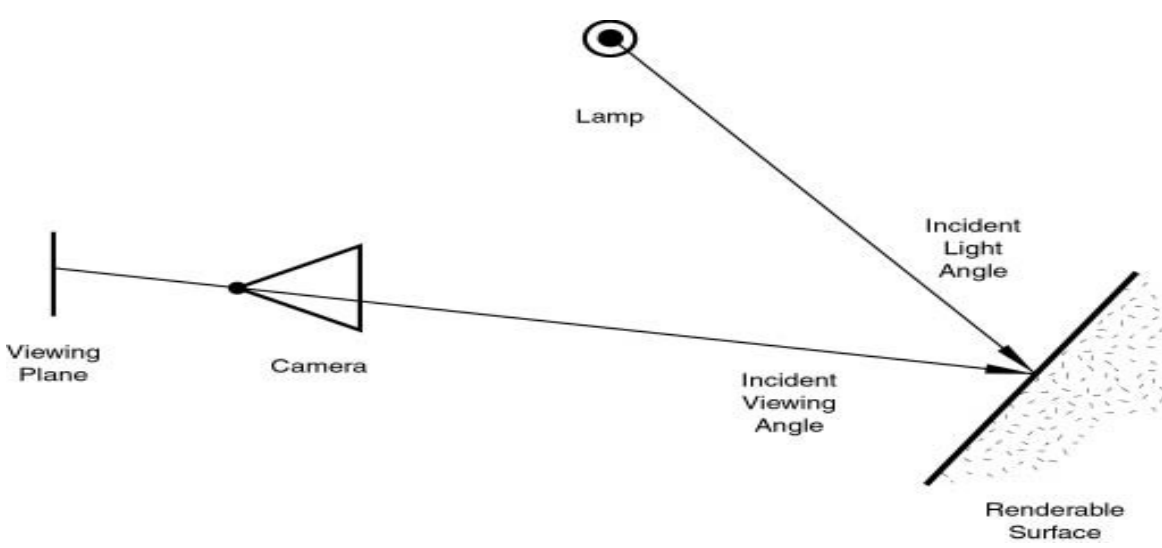

Figure 1. Rendering Engine Basic Principle

Two types of situation arises when at a pixel on given surface light falls, they are specular reflection and diffusion. The basic difference between diffusion and specular reflection is how striking light ray angle and reflected light ray is related to each other.

The shading of an object depends upon the base color of the object during rendering process and the intensity with which light is striking at the object.

\subsection{Textures}

It is a method by which we can add minute details to the given surface by projecting or focusing images on the object and some level of textures on the object surface. The images used for projection changes the color of the object, SRT (specular-reflection-transparency) of the object. Textures can be of applies to the various things such as material,world scene for creating clouds and natural sky color for the scene and to brush so they can work according to texture to be applied on the object.

Textures are the extra surface or layers on the object's base material of which it is made up of. The layers can be:

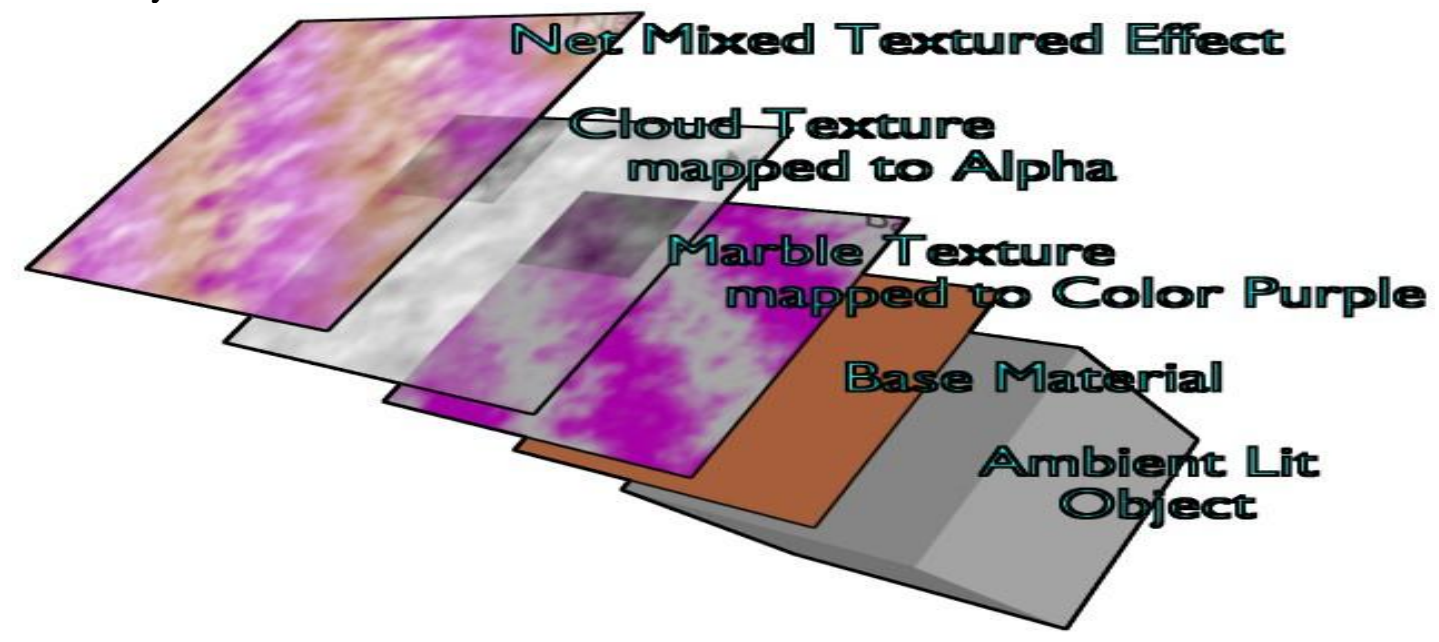

Figure 2. Layers of Texture for the Base Material

5.6.1. Object Texture: It can be defined as ambient light by which is lit up in the world view port. 
5.6.2. Base Material Texture: It is whole surface color which is spread uniformly and respond to light the way it strike and gives variety of shades of diffusion, mirror and speculars.

5.6.3. Primary Texture: This layer provides the effect of marble on the surface of the object. 5.6.4. Second Cloud Texture: It makes the surface like butter paper which is semi-opaque or semi-transparent, like misty or foggy.

5.6.5. Mixed Texture: Two texture are mixed with each other and then with base material texture so as to provide mixed texture effect.

The different files are used is that .blend, obj, .3ds, .lwo file, .brhfile, .rigged file, .bvh file.

\subsection{Constraints}

Constraints are a way of implementing relationship between the transformation properties like rotation, translation and scaling. Constraints are very useful in simple and static frames but they were designed initially for animation. They work as $4 \mathrm{X} 4$ matrices transformation internally. When scaling, rotation is used the value or the information is fetched from the $4 \mathrm{X} 4$ matrix only.

\subsection{Animation}

It can be thought as continuous displaying images different to make a sequence such that it gives view that object is translating or rotating or scaling. Human eyes cannot differ after 16 frames per second between images and considered it as single image which showing transformation. It can also be defined as an object that changes its size, position or shape over the period of time. Three methods are generally used for the transformation of the object in 3D space.

5.8.1. Key Frames: All the positions of the all objects are saved in units of time. Creation of animation involved interpolation of an object flowing through the frames. Need of this methods make to visualize the units of work. Animator moves the object from one position to another and inserting them into appropriate time of unit.

5.8.2. Animation Curves: Key frames allows at interpolate the curves and scaling, rotation and translation can be drawn for each XYZ component. The need of this method is when precise control of movement of an object is required.

5.8.3. Path: In 3D space drawing of curve takes place, and constrained are imposed on the objects and to follow these restrictions for given time function over the frames.

\subsection{Animation Techniques}

5.9.1. Classical 2D Animation: It is also known as hand drawn $2 \mathrm{D}$ animated sequence. For this type of animation animator required 12drawing on paper for one second of movie. For post-production drawing are captured by the computer. This technique was predominate until CGI came into picture.

5.9.2. Digital 2D Animation: This technique is come into picture for the use in television and web animation. Creation of frames take place directly by use of software. 
5.9.3. Digital 3D Animation: $3 \mathrm{D}$ characters are created which can be textured, rigged and animated in the world view port. It is one the best technique for the current scenario.

5.9.4. Stop-Motion Animation: Character is set in the desired position against the background to show the frame, and then modification is made to progress to another frame. The procedure is completed to make desired animation.

\subsection{Formatted Files}

5.10.1. Blend File: blender features is an internal file system that can pack multiple scenes into a single file called ".blend" file. All blender's blend files are forward, backward and cross platform compatible with other version of blender with the following reason: loading animation are stored in post-2.6 files in the blender and pre-2.6 .This is due to the reworked of the animation subsystem. The loading of meshes are stored in post 2.65 because of introduce of new concept BMESH, a more versatile and full mesh format. All formats of the objects, materials, textures, sounds, images, and post production effects for entire animation can be stored in a single format called "blend file". Here data are loaded from the other sources like images and sounds. Interface configurations are returned from the "blend file". It combines the different type of the data blocks like objects, meshes, lamps .an object meshes consists of various different type of the data blocks .this means when there is a polygon mesh consists of at least one object.

5.10.2. obj file:It is a geometric file format and it is used for advanced visualize animation package. The file format of OBJ file is opened and adopted by the graphics, and $3 \mathrm{D}$ animation. It is a universally accepted format. It looks simple data format that represent 3D geometry alone. This told about the position of each vertex, $\mathrm{u} v$ position of each texture, vertex of normal and the faces are made to polygon. Vertices are stored counter clockwise direction the OBJ coordinates has no units. It can contains the scale information and helps for human readable comment line.

5.10.3. 3ds File:It is one of the file format that used by Autodesk file format $3 \mathrm{ds}$ max $3 \mathrm{D}$ max modeling. Animation and rendering purposes, animation give more attractive to be human and rendering concept means to add color to model. 3D resources catalog along with .OBJ file which are more frequently used by the model as an archiving file format.

5.10.4. Iwo File:It is an object file format which is used by the light wave program. It is a programs which is used for 3D modeling, animation rendering. In the .LWO FILE objects are stored in format of the meshes and includes polygon, points, surfaces that is helpful for describe the models appearances. They are also referred the image file of texture when it required.

5.10.5. Rigged File: Rig is used for digital Skelton bound to the 3D mesh. RIG is made by joints and bones. Each behaves as a handle that makes the animator to bend the character in to a desired pose. RIG consists the process are placing the Skeleton, joint hierarchy, forward kinematics, inverse kinematics, degree of freedom, squash and stretch, facial rigging.

5.10.6. bvh File: it is used to capture the motion means motion of human model are first capture by help this file then that motion can be further used in 3D animation model .ASF 
and .AMC files are used capture the motion of model after that it convert to wildly used format like .BVH file format.

\section{Implementation}

Here the components are used to describe the story "3D ANIMATION FOR DRINK AND DRIVE" which are human, car, camera, and building.

For creating a human in 3D space first start with a cube. Model is symmetrically in nature so this concept applies in human modeling. Add a loop running vertically at the middle and delete the vertices on left, then add mirror modifier. After that add the loop horizontally and fashion out the torso. After that add a vertical loop on the torso so it makes more geometrically. Extract the head, leg and add head on the top, leg on bottom. After that extract the arms and then apply thumb and adjust the human model till it satisfied. For this modeling of human geometric primitives are used like polygon, curves, surfaces, cubes etc. and techniques are used is transformation, rotation, scaling etc. for coloring purposes rendering concept used. Human bones are made by rigged structure. Human walking from one to another that is capture by the $\mathrm{BVH}$ file.

Car is an object and design by help of the NURBS, blender, texture, mapping by path .NURBS surfaces are defined by the spline curves which are influenced and maintain by the weighted control points. Increasing the weight the point, pull the curve very closer to the point. These are smooth surfaces not using the flat surfaces. In this each have control point of control polygon. Sometimes polygons are too coarse-grained to use for a model so it fine grained surfaces are required that can be done by the texture mapping. It pastes the images on to the surfaces in the scene adding realistic fine detail without exploding the geometry.

For creating the tree fractal concept is used means repeated the same structure fractals are self-similar, complex higher dimension and gives infinite detail information at each and every points. Mainly it used for creating trees, mountains etc.

3D camera is used to the quickly create the motion and add depth of the animation. Actually assume camera is an object and it wants needs to react the environment. Suppose something passes near the camera it can react with camera. To handle simple move up-down right-left randomly every 10-13 frames and curves are splined in nature. Camera can used to correlate to view port. For more realistic image camera can used to the rotation purposes.

The multistoried building is created by the help of cube which is a primitive in blender. The cube is scaled to give a look of building and is colored by the help of blender tool material which gives it a realistic view. The building is actually a shopping mall with a bar inside it after which human comes out after having drink. Blender Render is used to show actual architecture of building.

\section{Result}

Following are the snapshots of the animation developed. 


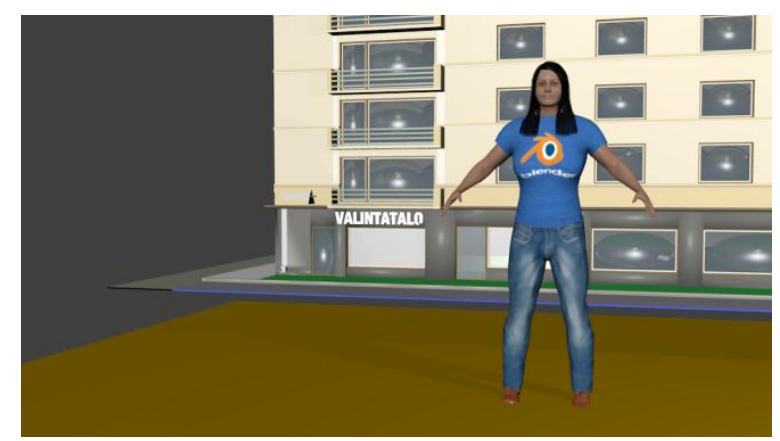

Figure 7.1. Development of Human using Make Human

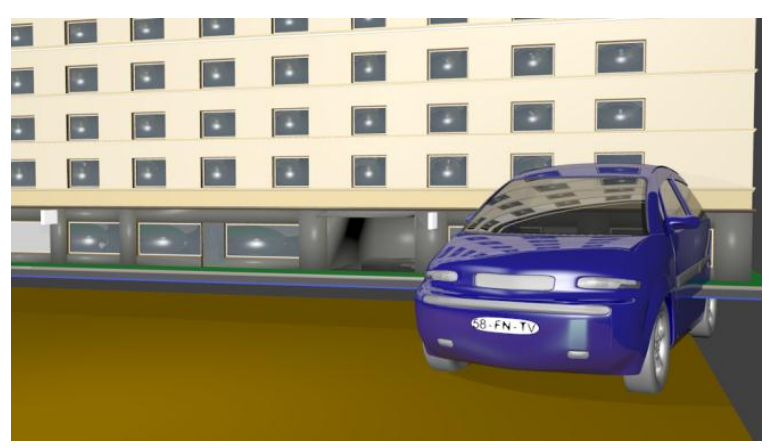

Figure 7.2. Development of Car using .Iwo File

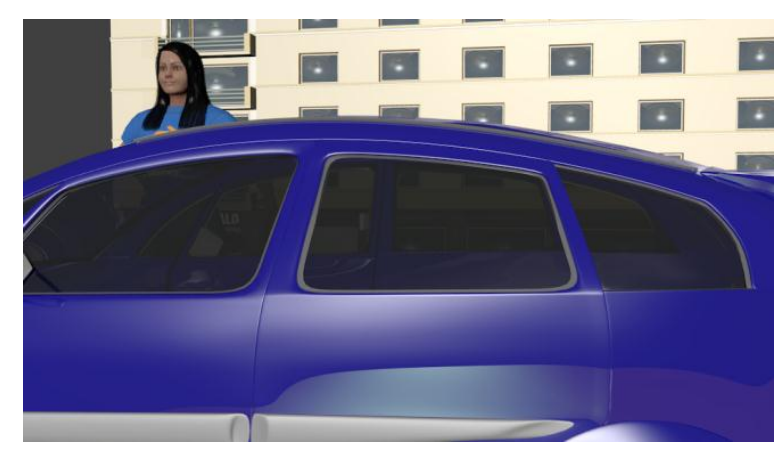

Figure 7.3. Human Sitting in Car

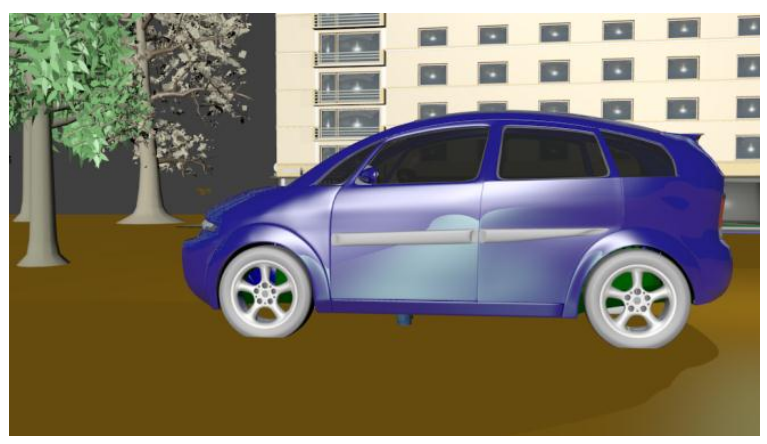

Figure 7.4. Still of Moving Car 


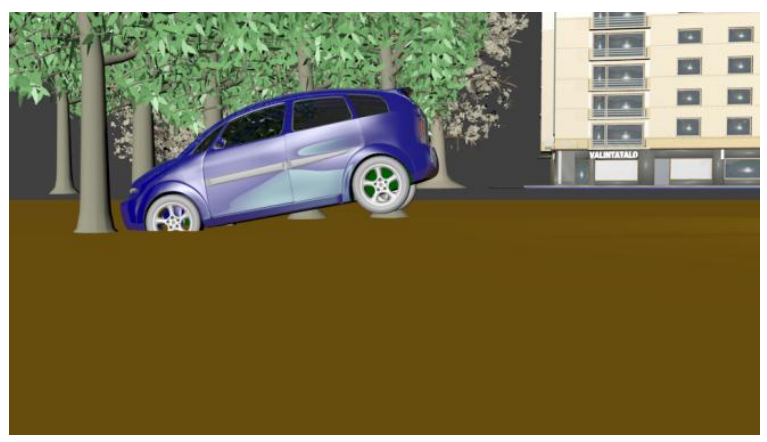

Figure 7.5. Car before Accident

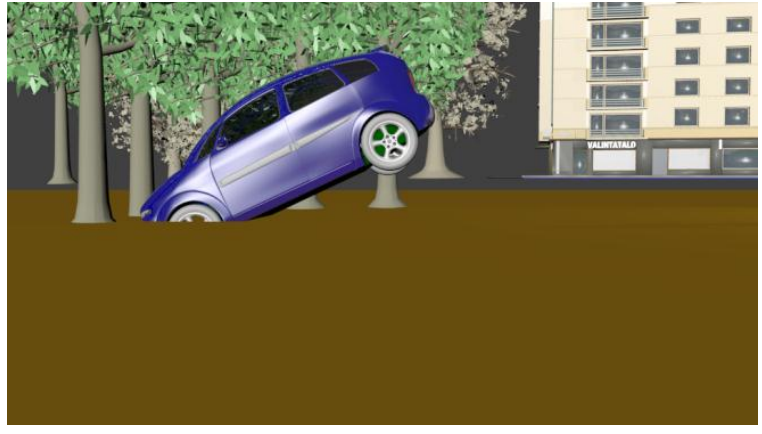

Figure 7.6. Car after Accident

\section{Conclusion}

This paper demonstrates 3D animation of drink and drive in which human is drunk and drives a car. The car meets with an accident .in this paper represent a human, a car, a tree, building by help of the make Human and blender software.

\section{Summary}

This paper is helpful in giving the message "Don't Drink and Drive" which is implemented using Blender and Make Human which are open source and Python based as they are freely available for commercial use and they also provide advantage in importing various formats of models. Limitation of this paper, it is not for visually impaired person as it lacks sounds. Future scope of this paper is to develop animation with sound and more number of frames and on a larger scale.

\section{References}

[1] Tai, Chu, Cohen and Lee, "Skeleton extraction", ACM Transactions on Graphics, vol. 27, no. 3, (2009).

[2] Ajay, Hetroy, Lazrus, and Depiraz, "Skeleton for character animation", In SCA '08: Proceedings of the 2008 ACM SIGGRAPH/Euro graphics Symposium on Computer Animation, (2008), pp. 151-160.

[3] Baran and Popovic, "Rigging and animation of 3D characters", ACM Transactions on Graphics, vol. 26, no. 3, (2008), p. 72.

[4] Blender-Foundation Blender3D (2008) www.blender.org.

[5] Brunner and Brunet, "Fast force field approximation and application to skeleton of discrete 3D objects". Computer Graphics Forum, Euro graphics 2010, vol. 27, no. 2, (2010), pp. 261-270. 
[6] Cornea, Silver, and Min, "Curve-skeleton properties and algorithms", IEEE Transactions on Computer Graphics, vol. 13, no. 3, (2010), pp. 530-548.

[7] Dellas, Moccozet, Magnenat-Thalmann, Mortara, Patane, Spagnuolo, and Falcidieno, "Extraction of control skeleton for animation", In SMI '09: Proceedings of the IEEE International Conference on Shape Modeling 2009, (2009), pp. 51-60.

[8] Demmel, Eisenstat, Gilbert, Li, and Liu. “A supermodel approach to partial pivoting” SIAM Journal on Matrix Analysis and Applications, vol. 20, no. 3, (2003), pp. 720-755.

[9] Hecker, Raabe, Enslow, DeWeese and Maynard, "Real-time retargeting to highly varied morphologies" In SIGGRAPH ’09: ACM SIGGRAPH 2009 Papers, (2009), pp. 1-11.

[10] Hilaga, Shinagawa, Kohmura, and Kunii, "Topology matching for similarity of 3D shapes", In SIGGRAPH05, (2005), pp. 203-212.

[11] Katz and Tal, "Hierarchical mesh decomposition using clustering and cuts", In SIGGRAPH '06: ACM SIGGRAPH 2006 Papers, (2006), pp. 954-961.

[12] Levy, OpenNL: "Open numerical library. alice.loria.fr/k.php/software/4-library/23-opennl.html”.

[13] Pascucci, Scorzelli, Bramer, and Mascarenhas, "Robust computation of graphs: simplicity and speed", ACM Transactions on Graphics, vol. 26, no. 3, (2009), p. 58.

[14] Poirier and Paquette, "Rig retargeting for 3d animation", (2006), http://profs.logti.etsmtl.ca/epaquette/Research/Papers/Poirier.2006/.

[15] Shapira, Shamir, and Cohen-Or, "Consistent mesh partitioning and skeleton using the shape diameter function”. The Visual Computer, vol. 24, no. 4, (2010), pp. 249-259.

[16] Tierny, Vandeborre, and Daoudi, "Enhancing 3D mesh skeletons with contour constrictions", The Visual Computer, vol. 24, no. 3, (2009), pp. 155-172.

[17] Wade and Parent, "Fast automated generation of skeletons for use in animation", In CA '02: Proceedings of the Computer Animation, p. 164 IEEE Computer Society, (2002).
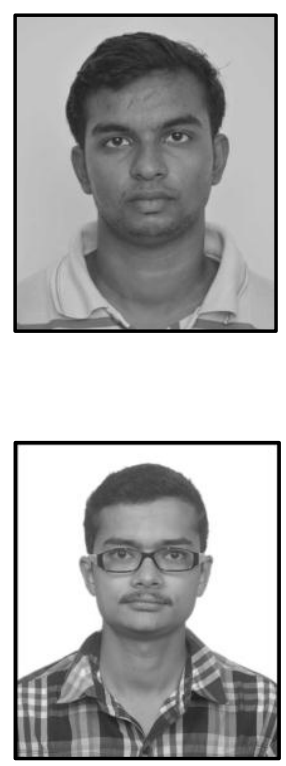

Imran Ahmed, $\mathrm{He}$ is currently pursuing post-graduation at VIT University Vellore,

Tamil Nadu in Computer Science and Engineering stream. He has done Bachelor of Technology in Computer Science and Engineering from University Institute of Engineering and Technology, CSJM University, Kanpur. His major interest work area is Computer Graphics, Cloud Computing, Artificial Intelligence and Web development.

Satish Janghel, He is currently pursuing post-graduation at VIT University Vellore,

Tamil Nadu in Computer Science and Engineering stream. He has done Bachelor of Engineering in Information Technology from Shri Shankaracharya College of Engineering and Technology, Bhilai. His major interest work area is Computer Graphics, Cloud Computing, Software Testing and Android development. 
International Journal of $u$ - and e- Service, Science and Technology Vol.8, No.1 (2015) 